\title{
COMPRESSION OF DATA COLLECTED IN BIG SCALE WIRELESS SENSOR NETWORKS
}

\author{
Gagan S Purad ${ }^{1}$ \\ ${ }^{I}$ Gagan S Purad, M.Tech,Computer Science Department, VIAT, Karnataka, India
}

\begin{abstract}
This paper introduces the first finish configuration to apply compressive testing hypothesis to sensor information gathering for largescale remote sensor systems. The fruitful plan created in this examination is relied upon to offer new edge of psyche for examination in both compressive testing applications furthermore, vast scale remote sensor systems. We consider the situation in which an expansive number of sensor hubs are thickly conveyed and sensor readings are spatially associated.The proposed compressive information social occasion has the capacity decrease worldwide scale correspondence cost without presenting serious calculation or convoluted transmission control.The heap adjusting trademark is fit for amplifying the lifetime of the whole sensor system and in addition person sensors. Moreover, the proposed plan can adapt to irregular sensor readings smoothly. We likewise complete the examination of the system limit of the proposed compressive information assembling and accept the examination through ns-2 recreations. All the more imperatively, this novel compressive information get-together has been tried on genuine sensor information and the outcomes demonstrate the effectiveness and power of the proposed plan.
\end{abstract}

KeyWords: Compressive Sampling, Wireless Sensor Networks

\section{INTRODUCTION}

This paper considers the information gathering issue in a substantial scale remote sensor system. Information gathering sensor system discovers a mixed bag of utilizations in foundation and natural surroundings observing [8][23]. It is normal that the quantity of sensor hubs conveyed could be on the request of hundreds or thousands. When all is said in done, information transmissions are refined through multi-jump directing from individual sensor hubs to the information sink. Effective arrangement of such expansive scale sensor systems confronts two noteworthy difficulties in compelling worldwide correspondence cost lessening and in vitality utilization burden adjusting.

The requirement for worldwide correspondence cost decrease is self-evident since such sensor organizes normally are created of hundreds to a huge number of sensors, creating huge measure of sensor information to be conveyed to information sink. It is all that much wanted to exploit the relationships among the sensor information to diminish the expense of correspondence. Existing methodologies receive in-system information pressure, for example, entropy coding or change coding, to lessen worldwide movement. On the other hand, these methodologies present huge calculation and control overheads that regularly not suitable for sensor systems applications.

The requirement for vitality utilization burden adjusting is likewise clear on account of the obliged multi-bounce information transmission for such extensive scale sensor systems. Fig. 1 shows such a system where sensors are thickly sent in the district of interest and screen nature all the time. A straightforward however run of the mill sample is the highlighted course in Fig. 1. This paper introduces the first finish configuration to apply compressive examining hypothesis [13][4][7] to sensor information gathering for extensive scale remote sensor systems (WSNs), effectively tending to the two noteworthy difficulties as delineated previously. To start with, the proposed information social occasion has the capacity accomplish generous sensor information pressure without presenting inordinate reckoning and control overheads. With exquisite configuration, the proposed plan is likewise ready to scatter the correspondence expenses to all sensor hubs along a given sensor information social occasion course. This will bring about a characteristic burden adjusting and augment the lifetime of the sensor system.

The fundamental thought of the proposed compressive information gathering (CDG) is delineated in Fig. 2(b). Rather than getting person sensor readings, the sink will be sent a couple weighted entireties of the considerable number of readings, from which to restore the first information. To transmit the ith whole to the sink, s1 increases its perusing d1 with an arbitrary coefficient $\varphi$ i1 and sends the item to s2. After accepting this message, s2 increases its perusing d2 with an arbitrary coefficient $\varphi \mathrm{i} 2$ and afterward sends the entirety $\varphi \mathrm{i} 1 \mathrm{~d} 1+\varphi \mathrm{i} 2 \mathrm{~d} 2$ to $\mathrm{s} 3$. Likewise, every hub sj adds to the handed-off message by including its own particular item. At last, the sink gets $\mathrm{N} \mathrm{j}=1$ pijdj, a weighted whole of the considerable number of readings. This procedure is rehashed utilizing $\mathrm{M}$ sets of distinctive weights so that the sink will get $\mathrm{M}$ weighted to. 


\section{RELATED WORK}

The fundamental assumption of in-network data compression is that sensor nodes have spatial correlations in their readings. According to where the spatial correlation is utilized, we can classify existing in-network data compression techniques into two categories.

\subsection{Conventional Compression}

Traditional pressure systems use the connection amid the encoding process and require express information correspondence among sensors.Cristescu et al. [12] propose a joint entropy coding methodology, where hubs utilization handed-off information as side data to encode their readings. Once more take the multi-jump course in Fig. 2 as an illustration. In the first place, hub s1 encodes its perusing d1 into message p1 utilizing $\mathrm{H}(\mathrm{d} 1)$ bits, where $\mathrm{H}(\mathrm{d} 1)$ is the entropy of $\mathrm{d} 1$. At that point, when $\mathrm{s} 2$ gets $\mathrm{p} 1$, it encodes its perusing $\mathrm{d} 2$ into message $\mathrm{p} 2$ utilizing $\mathrm{H}(\mathrm{d} 2 \mid \mathrm{d} 1)$ bits, where $\mathrm{H}(\mathrm{d} 2 \mid \mathrm{d} 1)$ is the restrictive entropy. Since $\mathrm{d} 1$ and $\mathrm{d} 2$ are associated, $\mathrm{H}(\mathrm{d} 2 \mid \mathrm{d} 1)$ is littler than $\mathrm{H}(\mathrm{d} 2)$. Consequently, mutually encoded messages cost less bits than freely encoded messages.

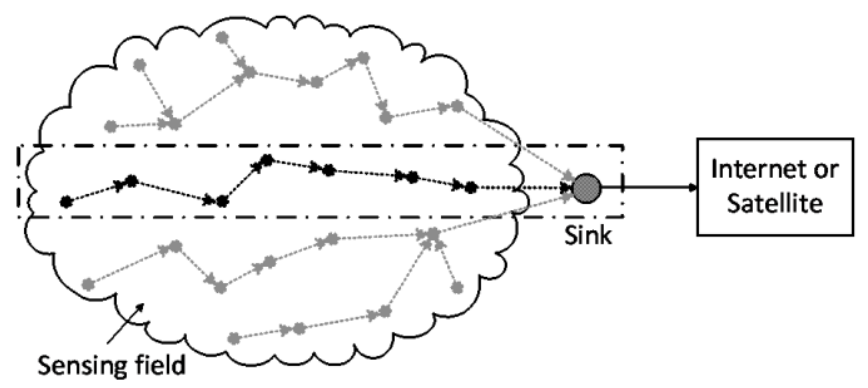

Figure 1: Gathering data in Sensor Network

The above methodology uses information relationship just unidirectionally. On the off chance that information are permitted to be imparted back furthermore, forward amid encoding, hubs might agreeably perform change to better use the connection. Ciancio et al. [10] and A'cimovi'c et al. [2] propose to pack piecewise smooth information through disseminated wavelet change. In doing as such, even hubs first show their readings. After accepting the readings from both sides, odd hubs figure the high pass coefficients $h(\cdot)$. At that point, odd hubs transmit $\mathrm{h}(\cdot)$ back and even hubs figure the low pass coefficients 1(.). This procedure is represented in Fig. 3. Despite the fact that wavelet decorrelationcan be performed for different levels, it is most certainly not proposed to do as such in circulated preparing on account of the correspondence overhead. After the change, hubs transmit critical coefficients to the sink, generally in their crude structure to maintain a strategic distance from the many-sided quality of entropy coding.

There are two primary issues with customary pressure methods. In the first place, the pressure execution depends vigorously on how the courses are sorted out. To accomplish the most astounding pressure proportion, pressure and steering calculations should be mutually improved. This has been demonstrated to be a NP-hard issue [12]. Second, the productivity of anin-system information pressure plan is not singularly decided by the pressure proportion, additionally relies on upon the computational what's more, correspondence overheads. Notwithstanding, joint entropy coding procedures perform complex reckoning in sensors, while change based procedures oblige a vast measure of information trades.

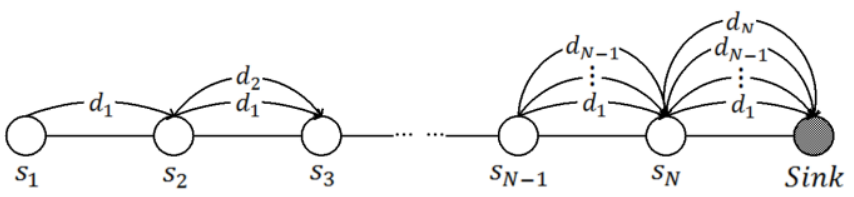

(a) Baseline data collection

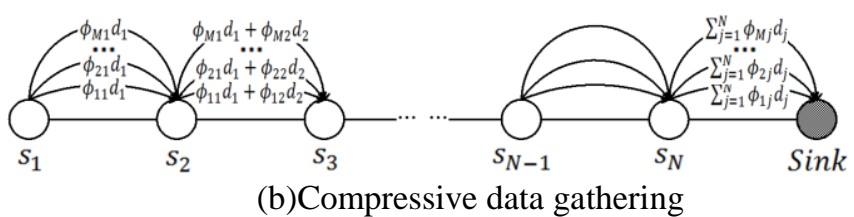

Figure 2: Comparing baseline data collection and compressive data gathering in a multi-hop route

\subsection{Distributed Source Coding}

Dispersed source coding systems [9][11][18] plan to lessen many-sided quality at sensor hubs and use connection at the sink. They are taking into account the Slepian-Wolf coding hypothesis [22], which guarantees that pressure of corresponded readings, at the point when independently encoded, can accomplish same effectiveness as though they are mutually encoded, if that messages are together decoded. This imperative conclusion not just wipes out information trades, yet decouples steering from pressure. In the wake of encoding sensor readings freely, each hub basically sends the packed message along the most brief way to the sink. Then again, an essential of Slepian-Wolf coding is that the worldwide connection structure should be known with a specific end goal to dispense fitting number of bits to be utilized by every hub. This is difficult to satisfy in an expansive scale remote sensor system.

In perspective of this, Yuen et al. [27] receives a restricted Slepian- Wolf coding plan. Taking into account the suspicion that sensors outside quick neighborhood have powerless relationship in their readings, a hub might just consider its information connection with one-bounce neighbors when deciding the measure of encoded message. We will demonstrate that, for an arrangement of genuine sensor information which don't fulfill this supposition, the confined coding plan will neglect to pack such information.

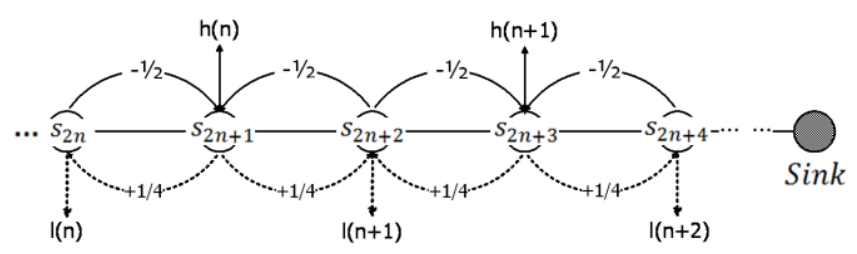

Figure 3: Cooperative wavelet compression 
With the development of compressive testing hypothesis [13] [4] [7], we have seen another street of examination in the field of in-system information pressure. Compressive remote detecting (CWS) [3] has all the earmarks of being ready to decrease the inertness of information delivering so as to gather in a solitary jump system direct projectionsof sensor readings through synchronized amplitudemodulatedsimple transmissions. Because of the troubles in simple synchronization, CWS is less reasonable for vast scale sensor systems. Rabbat et al. [21] influences compressive inspecting for information tirelessness, rather than information gathering, in a WSN. In an outline paper, Haupt et al. [17] additionally theorize the capability of utilizing compressive testing hypothesis for information total in a multi-bounce WSN. Be that as it may, no genuine plan has been accounted for in light of this beginning thought. At the point when compressive testing is connected to in-system information pressure, it will bring an abundance of comparable advantages as conveyed source coding including straightforward encoding procedure, sparing of between hub information trade, and decoupling of pressure from directing. Besides, compressive inspecting has two extra preferences. Initially, it can manage strange sensor readings nimbly. This favorable position will be point by point in the following segment. Second, information reproduction is most certainly not touchy to parcel misfortunes. In compressive inspecting, all messages gotten by the sink are just as vital. On the other hand, in appropriated source coding, got information are predefined as primary or side data. Losing fundamental data will cause deadly lapses to the decoder. All these sought benefits make compressive examining a promising answer for the information gathering issue in substantial scale remote sensor systems.

\section{COMPRESSIVE DATA GATHERING}

The goal of compressive information social affair is twofold: pack sensor readings to lessen worldwide information movement and disperse vitality utilization equitably to drag out system lifetime. Like disseminated source coding, the information relationship design might be used on the decoder end. Also, pressure and steering are decoupled and subsequently can be independently upgraded.

\subsection{Data Gathering}

The instinct behind CDG is that higher effectiveness can be accomplished if related sensor readings are transmitted mutually instead of independently. We have given a basic case in Segment I, demonstrating how sensor readings are consolidated while being handed-off along an anchor sort topology to the sink. In rehearse, sensors as a rule spreads in a two-dimensional zone, what's more, the outfit of directing ways shows a tree structure. Fig. 4(a) demonstrates a regular directing tree in which the sink has four youngsters. Each of them leads a subtree delimited by the dabbed lines. Information social affair and remaking of $\mathrm{CDG}$ are performed on the subtree premise.

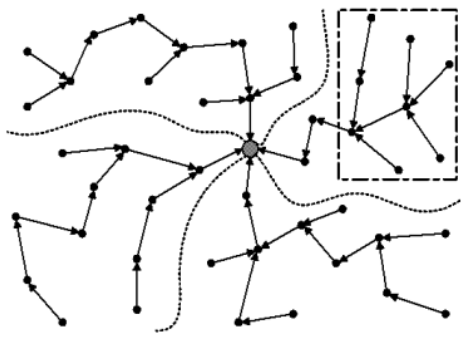

(a)

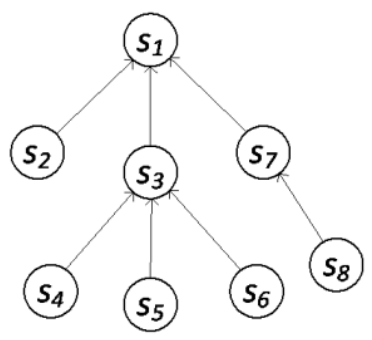

(b)
Figure 4: Gathering of data

With a specific end goal to join sensor readings while transferring them, each hub needs to know its nearby steering structure. That is, regardless of whether a given hub is a leaf hub in the steeringtree or what number of youngsters the hub has on the off chance that it is an internal hub. To encourage productive collection, we have made a little change to standard specially appointed steering convention: at the point when a hub picks a guardian hub, it sends a "subscribe notice" to that hub; when a hub changes guardian, it sends an "unsubscribe notice" to the old guardian.

The information social occasion procedure of CDG is shown through a sample demonstrated in Fig. 4(b). It is the nitty gritty perspective of a little division of the steering tree stamped in Fig. 4(a). After all hubs obtain their readings, leaf hubs start the transmission. In this sample, s2 produces an arbitrary number $\varphi \mathrm{i} 2$, figures $\varphi \mathrm{i} 2 \mathrm{~d} 2$, and transmits the quality to s1. The file $\mathrm{i}$ means the ith weighted whole running from 1 to $M$. Essentially, s4, s5 and s6 transmits $\varphi \mathrm{i} 4 \mathrm{~d} 4, \varphi \mathrm{i} 5 \mathrm{~d} 5$, and $\varphi \mathrm{i} 6 \mathrm{~d} 6$ to $\mathrm{s} 3$. When s3 gets the three qualities, it figures $\varphi \mathrm{i} 3 \mathrm{~d} 3$, adds it to the entirety of transferred values and transmits $6 \mathrm{j}=3$ pijdj to $\mathrm{s} 1$. At that point $\mathrm{s} 1$ processes $\varphi \mathrm{i} 1 \mathrm{~d} 1$ and transmits $8 \mathrm{j}=1 \varphi 1 \mathrm{jdj}$. At long last, the message containing the weighted aggregate of all readings in a subtree is sent to.

\subsection{Data Recovery}

As indicated by compressive inspecting hypothesis, a Kmeager sign can be reproduced from a little number of estimations with a likelihood near one. The weighted entireties acquired in (2) are a regular kind of estimations. Signal sparsity describes the relationships inside of a sign. A $\mathrm{N}$-dimensional sign is considered as a $\mathrm{K}$-inadequate sign on the off chance that there exists a space in which this sign can be spoken to by $(\mathrm{K} \mathrm{N})$ non-zero coefficients. Fig. 5(a) demonstrates a 100-dimensional sign in its unique time area. Clearly, it is not scanty at all in this space. In light of the sign relationship, it can be portrayed all the more minimally in change spaces, for example, wavelet and DCT. Fig. 5(b) gives the representation of the same sign in DCT space. We can see that there are just 5 non-zero DCT coefficients. Along these lines, this sign is a 5-inadequate sign in DCT space. 


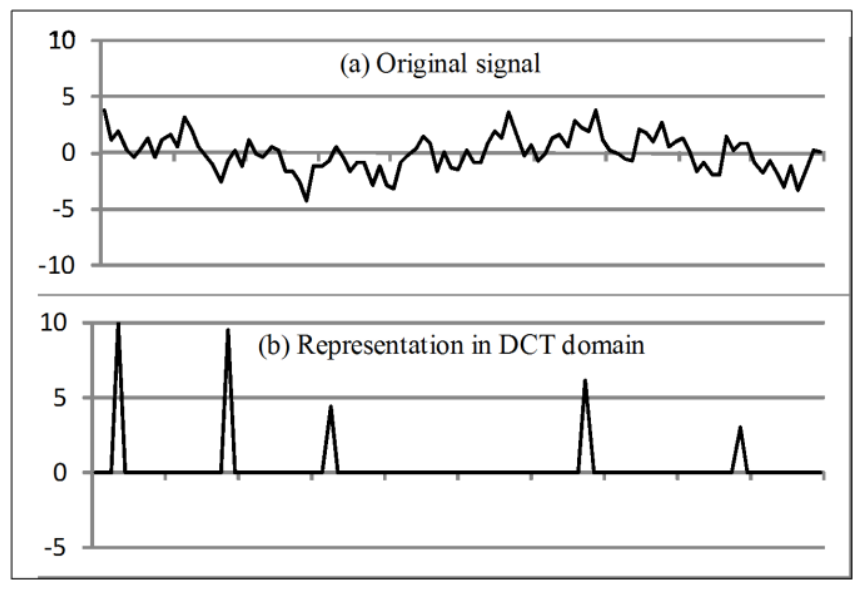

Figure 5: DCT domain with 5 Sparse signal

\section{NETWORK CAPACITY OF \\ COMPRESSIVE DATA GATHERING}

The past area outlined how to assemble and recoup sensor readings obtained in one time occurrence. This area will research the advantage of CDG from the perspective of system limit, i.e. how visit CDG permits sensors to get information while guaranteeing all readings can be transmitted to the sink. The limit of an information gettogether system is characterized as takes after. Definition 1 (System Limit). We should characterize that a rate $\lambda$ is achievable in an information gathering sensor system, on the off chance that there exists a period occasion $\mathrm{t} 0$ and term $\mathrm{T}$ such that amid $[\mathrm{t} 0, \mathrm{t} 0+\mathrm{T})$ the sink gets $\lambda \mathrm{T}$ bits of information created by each of the sensors si, $i=1,2, \ldots N$. At that point, system limit $\mathrm{C}$ is characterized as the supremum of the achievable rate, on the other hand $C=\sup \{\lambda\}$. Unique in relation to the spearheading deal with system limit examination [16], the activity design in our study is numerous to-one. We let all sensors create information at the same rate, and expect that sensor readings gained in the meantime occasion are K-scan.

\subsection{NS 2 Simulations}

The system limit investigation is in view of planned medium access control (Macintosh). Practically speaking, the computational and correspondence overhead of Macintosh planning is too high. Controversy based Macintosh is all the more frequently embraced in remote sensor systems. So as to see how CDG performs in down to earth settings, we assess its execution through ns- 2 [20] reenactments and contrast it and pattern transmission on two normal topologies: chain [8] and lattice topologies [24]. Table 1 rundowns the principle parameters utilized as a part of the reproduction. We receive 802.11 rather than ZigBee on the grounds that the usage of 802.11 in ns- 2 is entrenched. For effortlessness, we will investigate the bundle rate of bit rate. Every parcel contains one and only message which is thought to be 20 bytes for both pattern transmission furthermore, CDG. Albeit both methodologies can join numerous messages in one parcel and enhance transmission productivity, we don't utilize expansive parcels in light of the fact that we are just intrigued in the examination between them. Information sparsity is accepted to be $5 \%$. For instance, when $\mathrm{N}=1000, \mathrm{~K}=50$, andwe expect that the sink can recoup the first information from $M=200$ irregular estimations. In the best case, CDG should accomplish limit increase of $\mathrm{N} / \mathrm{M}=5$.

\subsection{Chain Topology}

The chain topology is made out of 1000 sensors and one sink situating at one amazing of the chain. The separation between any two neighboring hubs are 10 meters. Under the given transmission and obstruction range, hubs can just speak with contiguous hubs, and may bring about meddle to two-bounce neighbors. In the recreation, we change the information interim and assess how yield interim and bundle misfortune proportion change as needs be. All in all, as the info interim abatements, the yield interim diminishes and the parcel misfortune proportion increments. In any case, on the off chance that an information interim is not achievable, the yield interim will stop to reduction and may marginally increment thus of blockage breakdown. We may surmise the system limit from the base accomplished yield interim. Fig. 6(a) demonstrates that the base yield interim of gauge transmission is 10.6 seconds for every message, and it is accomplished at the point when the information interim is 10.2 seconds for every message. There is a little crevice between these two qualities in view of system nerves and parcel misfortunes. Fig. 6(b) demonstrates the execution of CDG. The base yield interim is 2.11 second for every message accomplished when the info interim is 1.92 second for every message. We can see that CDG can accomplish a limit pick up of 5 over pattern transmission.

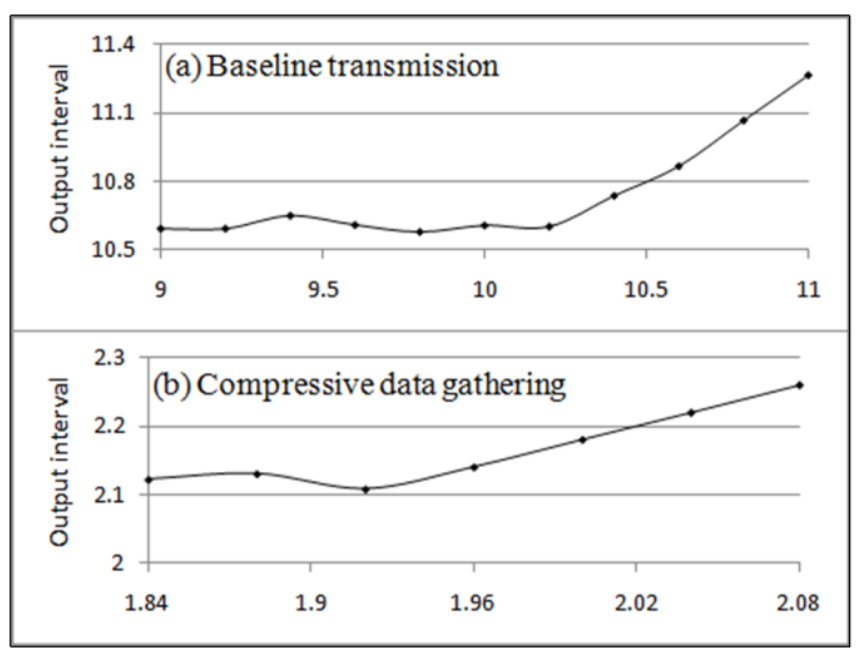

Figure 6: Interval of input-output in chain topology

\section{CONCLUSION AND FUTURE WORK}

We have depicted in this paper a novel plan for vitality effective information gathering in expansive scale remote sensor systems in light of compressive inspecting hypothesis. We accept this is the first finish configuration to change over the customary pack then-transmit process into a compressive social affair (pack with-transmission) procedure to address the two noteworthy specialized difficulties that today's huge scale sensor systems are 
confronting. In the advancement of the proposed plan, we have completed the examination of limit for remote sensor system when compressive information gathering is embraced. We have demonstrated that CDG can accomplish a limit addition of N/M over pattern transmission. We have too outlined ns-2 reproductions to accept the proposed planat the point when discord based Macintosh is utilized. Moreover, numerical studies taking into account genuine sensor information not just confirmed information sparsity in handy information securing, additionally illustrated the proficiency and vigor of the sensor information remaking with and without strange readings. It ought to be noticed that effective utilization of CDG depends on the properties of sensor field. On the off chance that sensor readings are not inadequate in any known area and in any legitimate request, CDG can't accomplish limit pick up in light of the fact that a vital essential of compressive inspecting hypothesis is absent. At the other great, when detecting information are meager in the first space, i.e. just a little part of sensors secure nonzero readings, it would be more effective to specifically transmit these non-zero readings through multibounce sending. CDG is not suitable for little scale sensor systems when signal sparsity may not be sufficiently noticeable and the potential limit addition may be too little. CDG is additionally more successful for systems with stable directing structure. This is since incessant hub disappointment or element course change will lead to high control overhead that possibly counteract the pick up from information pressure. We are as of now exploring the expansion of CDG to all the more difficult systems administration situations what's more, the abuse of adaptation to non-critical failure of the compressive testing standards to accomplish more powerful execution in sensor information gathering.

\section{REFERENCES}

[1] NBDC CTD data. http://tao.noaa.gov/refreshed/ctd delivery.php.

[2] J. A'cimovi'c, B. Beferull-Lozano, and R. Cristescu. Adaptive distributed algorithms for power-efficient data gathering in sensor networks. In Proc. of Intl. Conf. on Wireless Networks, Comm. and Mobile Computing, pages 946-951, Jun. 2005.

[3] W. Bajwa, J. Haupt, A. Sayeed, and R. Nowak. Compressive wireless sensing. In Proc. of IPSN, pages 134-142, Apr. 2006.

[4] R. Baraniuk. Compressive sensing. IEEE Signal Processing Magazine, 24(4):118-121, Jul. 2007.

[5] T. Blumensath and M. E. Davies.Gradient pursuits. IEEE Trans. on Signal Processing, 56(6):2370-2382, Jun. 2008.

[6] E. Candes, J. Romberg, and T. Tao. Robust uncertainty principles: Exact signal reconstruction from highly incomplete frequency information. IEEE Trans. Inform. Theory, 52(2):489-509, Feb. 2006.

[7] E. J. Cand'es and M. B. Wakin. An introduction to compressive sampling. IEEE Signal Processing Magazine, 25(2):21-30, Mar. 2008.

[8] C. W. Chen and Y. Wang. Chain-type wireless sensor network for monitoring long range infrastructures: architecture and protocols. International Journal on Distributed Sensor Networks, 4(4), Oct. 2008.

[9] J. Chou, D. Petrovic, and K. Ramchandran. A distributed and adaptive signal processing approach to reducing energy consumption in sensor networks. In Proc. of IEEE Infocom, pages 1054-1062, Mar. 2003.[4]. Reference 4

\section{BIOGRAPHIES}

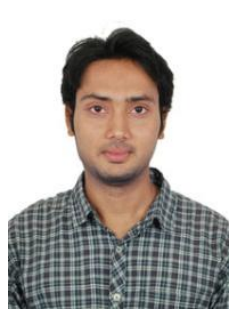

Gagan S Purad

Pursuing M.Tech Final year

VIAT Bangalore 